BMJ Open Ophthalmology

\section{Outcomes of pars plana vitrectomy with subretinal tissue plasminogen activator injection and pneumatic displacement of fovea-involving submacular haemorrhage}

To cite: Wilkins CS, Mehta N, Wu CY, et al. Outcomes of pars plana vitrectomy with subretinal tissue plasminogen activator injection and pneumatic displacement of fovea-involving submacular haemorrhage. BMJ Open Ophthalmology 2020;5:e000394. doi:10.1136/ bmjophth-2019-000394

Association for Research in Vision and Ophthalmology Annual Meeting, 20 April 2019, Vancouver, Canada; American Society of Retina Specialists Annual Meeting, July 212019 , Chicago, Illinois.

Received 14 September 2019 Revised 13 January 2020 Accepted 19 February 2020
Check for updates

\section{(c) Author(s) (or their} employer(s)) 2020. Re-use permitted under CC BY-NC. No commercial re-use. See rights and permissions. Published by BMJ.

New York Eye and Ear Infirmary of Mount Sinai, New York, New York, USA

\section{Correspondence to} Dr Carl S Wilkins; cswilkins013@gmail.com

\section{ABSTRACT}

Objective Fovea-involving subretinal haemorrhage is challenging to manage with uncertain visual outcomes. We reviewed outcomes of patients with fovea-involving macular haemorrhage treated with pars plana vitrectomy (PPV) and subretinal tissue plasminogen activator (tPA) with pneumatic displacement.

Methods and Analysis This is a retrospective interventional case series. All patients with submacular haemorrhage who underwent PPV with subretinal tPA injection were included. Reasons for exclusion encompassed patients who underwent intravitreal tPA injection in the office without surgery, insufficient followup or documentation. Primary outcomes of interest were postoperative visual acuity (VA) at month 1 and 3. Secondary outcomes were median VA at month 3 by location of haemorrhage and underlying diagnosis.

Results Thirty-seven total patients were included. The mean age was 68.2 years, with $54.1 \%$ (20/37) females. The most common aetiology was exudative macular degeneration (43.2\%), followed by undifferentiated choroidal neovascularisation (CNV) (18.9\%), polypoidal choroidal vasculopathy (18.9\%), traumatic CNV (10.8\%), macroaneurysm $(5.4 \%)$ and proliferative diabetic retinopathy (2.7\%). Median preoperative VA was $20 / 2000$, postoperative month 1 was $20 / 347(p<0.01)$, improving to $20 / 152(p<0.01)$ at month 3. Proportion of patients gaining vision $3+$ lines in vision was 15/36 (42\%). Mean preoperative central subfield thickness on optical coherence tomography was $512.2 \mu \mathrm{m}$ for sub-retinal pigment epithelium haemorrhage and $648.2 \mu \mathrm{m}$ for subretinal haemorrhage $(p=0.48)$. Difference in VA by diagnosis was not significant $(p=0.60)$.

Conclusions PPV with subretinal tPA injection and pneumatic displacement of submacular haemorrhage offers modest visual recovery for a diverse group of patients. Location of haemorrhage or specific diagnosis may not predict outcome.

\section{INTRODUCTION}

Fovea-involving subretinal macular haemorrhage (SRMH) is a vision-threatening ocular condition with a diverse group of underlying

\section{Key messages}

What is already known? Subretinal macular haemorrhage (SRMH) has been shown to have poor natural history outcomes, with similar prior interventional series suggesting benefit of pars plana vitrectomy (PPV) and subretinal tissue plasminogen activator (tPA) in patients with exudative macular degeneration.

What are the new findings?

This study comprises the most diverse patient cohort reported of SRMH treated with PPV and subretinal tPA, with no significant difference in outcome found between subgroups

The anatomic location of haemorrhage on optical coherence tomography, whether predominately subretinal or sub-retinal pigment epithelium, was not shown to prognosticate visual outcome.

$>$ How might these results change the focus of research or clinical practice? The underlying pathogenesis of SRMH was not shown to correlate with visual outcome after surgery, suggesting potential benefit of intervention regardless of underlying disease. The decision to operate should not be made based upon anatomic location of hemorrhage, but rather considered on a case-by-case basis depending on medical stability for surgery, possibilty of post-operative positioning, and patient goals.

causes. Experimental models have documented the toxicity of subretinal blood, with early retinal photoreceptor disintegration and subsequent cell death. ${ }^{1}$ In addition to direct toxicity, the blood clot has been shown to result in fibrin-mediated tractional damage within the retinal laminations. ${ }^{2}$ Additionally, natural history studies have demonstrated large variation in visual recovery, ranging from return of baseline best corrected visual acuity (BCVA) to loss of 6 lines or more in visual acuity (VA). ${ }^{34}$ Patients with SRMH secondary to underlying choroidal neovascularisation 
$(\mathrm{CNV})$ tend to do worse without intervention, while those secondary to trauma, valsalva retinopathy, or acute vascular occlusion may have a better prognosis. ${ }^{4}$

Given the high risk of visual decline following SRMH involving the fovea, attention has been directed towards interventions which can potentially prevent or attenuate vision loss following an acute event. Previous retrospective case series of intravitreal anti-vascular endothelial growth factor (VEGF) therapy, alone or in combination with tissue plasminogen activator (tPA) and gas tamponade, suggest some treatment benefit if administered shortly following acute SRMH. ${ }^{56}$ Several groups have investigated the role of pars plana vitrectomy (PPV) with tPA injection and air-fluid displacement for thick SRMH. Early retrospective studies published promising results in patients with SRMH due to exudative age-related macular degeneration (AMD), with statistically significant improvement in VA, successful anatomic displacement and relatively few serious complications. ${ }^{78}$ Chang et al published a large, multicentre retrospective series of patients with exudative AMD and SRMH, demonstrating successful anatomic result in $82 \%$ of patients, with modest improvement in visual recovery postoperatively, greatest in patients with CNV who continued anti-VEGF injections at follow-up. ${ }^{9}$ Recent studies in patients with AMD, polypoidal choroidal vasculopathy (PCV) and other causes of SRMH have promoted the addition of air in the tPA injected to enhance displacement of coagulated blood. ${ }^{1011}$

This study is a retrospective, interventional case series of all patients with fovea-involving, dense SRMH who underwent PPV with subretinal tPA injection and pneumatic displacement. We chose to take a universal approach to our evaluation by including all patients regardless of underlying pathophysiology, to derive a more generalised answer to expected visual outcome, and potential complications from this treatment approach.

\section{MATERIALS AND METHODS}

Patient and public Involvement

Patients were not directly involved in the acquisition, analysis, or interpretation of this data.

\section{Methods}

A review of hospital records was performed of all patients with any billing code including PPV who also had an associated pharmacy J-code for intraoperative tPA linked to the same encounter. Institutional review board (IRB) approval was obtained. All patients undergoing treatment had thick SRMH involving the fovea, the thickness being defined as perceptible elevation of retina on biomicroscopy or as distortion of foveal contour on optical coherence tomography (OCT). Full informed consent was obtained and documented from all patients prior to surgery. IRB approval was obtained for retrospective chart review. The dose of subretinal tPA was 25-50 mcg, with addition of balanced salt solution, subretinal antiVEGF and volume of subretinal air at the discretion of the individual surgeon. Primary outcomes included VA at 1-month and 3-month follow-up visits, as well as surgicallyrelated complications. Secondary outcomes included BCVA related to anatomic location of haemorrhage on OCT and BCVA related to underlying diagnosis. Patients were evaluated at 1-month and 3-month visit intervals, which were determined using contiguous interval windows, categorising the visit by rounding up or down to the closest follow-up interval by the mid-point between visits. Patients were excluded if they received intravitreal tPA injection and gas in the office without surgery, if lost to follow-up, or if operative documentation was lacking despite a linked pharmacy code. Spectral domain OCT data were obtained for all patients when possible. The location of haemorrhage was divided into predominantly sub-retinal pigment epithelium (RPE) $(>50 \%)$ or predominantly subretinal $(>50 \%)$ based on peak height OCT imaging measured manually by investigators using the calliper tool function from internal limiting membrane (ILM) to nearest RPE. Median preoperative, postoperative month 1 and month 3 Snellen VAs were recorded and were analysed using Wilcoxon paired signed-rank test (SPSS V.22.0 software). All VAs were converted into LogMAR for analysis, with hand motion vision given a value of 3.0. Due to the inability to assign discrete numerical acuity values, patients with light perception (LP) or worse vision were excluded from interval analysis. Proportions of patients gaining one or more and three or more lines in vision were calculated for the 3-month interval visit. Last observation carried forward was used for 3-month interval analysis when appropriate. Median vision between underlying diagnoses was compared using one-way analysis of variance (ANOVA).

\section{RESULTS}

We identified 37 who fit criteria and had sufficient follow-up data. The baseline characteristics are presented in table 1.

The most common underlying diagnosis was wet AMD, followed by undifferentiated CNV and PCV. Eight patients $(21.6 \%)$ were receiving anticoagulation or antiplatelet therapy at the time of acute SRMH. Mean time from acute SRMH to surgical intervention was 14.6 days (median 11 days, range 2-63 days). Successful anatomic displacement of SRMH was achieved in 25/28 (89.3\%) patients with available postoperative OCT measurements. figures 1 and 2 show a representative case of displacement. One of the three failed displacements was due to early re-bleed. Eleven patients $(29.7 \%)$ experienced complications including five vitreous haemorrhages $(13.5 \%)$, four recurrent submacular haemorrhages $(10.8 \%)$, three rhegmatogenous retinal detachments $(8.1 \%)$, one bloodstained cornea $(2.7 \%)$, one glaucoma $(2.7 \%)$ and one progression to phthisis (2.7\%). Twenty-nine patients $(78.4 \%)$ received co-application with anti-VEGF injection (bevacizumab) at time of surgery.

Median pre-operative VA was 20/2000 (2.00 LogMAR). Median VA at post-op month 1 was 20/347 (1.24 LogMAR; 


\begin{tabular}{ll}
\hline Table 1 Baseline characteristics & \\
\hline Characteristic & Value \\
\hline Mean age (range) & $68.2(24-93)$ \\
\hline Female N (\%) & $20(54.1)$ \\
\hline Diagnosis N (\%) & \\
wAMD & $16(43.2)$ \\
Undifferentiated CNV & $8(21.6)$ \\
\hline IPCV & $7(18.9)$ \\
\hline Trauma/CNV & $3(8.1)$ \\
\hline Macroaneurysm & $2(5.4)$ \\
\hline PDR & $1(2.7)$ \\
\hline Phakic status N (\%) & \\
\hline Pseudophakic & $21(56.8)$ \\
\hline Phakic & $15(40.5)$ \\
\hline Aphakic & $1(2.7)$ \\
\hline
\end{tabular}

CNV, choroidal neovascularisation; IPCV, idiopathic polypoidal choroidal vasculopathy; PDR, proliferative diabetic retinopathy; WAMD, wet age-related macular degeneration.

$\mathrm{p}<0.01)$ improving to $20 / 152(0.88 \log M A R ; p<0.001)$ at month 3 (table 2).

Overall, 22/36 patients $(61.1 \%)$ experienced visual improvement post-operatively, with $15 / 36$ patients $(41.7 \%)$ gaining $3+$ lines and seven patients (18.9\%) gaining 1-2 lines of improvement. Five patients experienced worsening of vision. Median VA showed statistical improvement at month 3 for patients with PCV $(\mathrm{p}=0.043)$ and with traumatic $\mathrm{CNV}$ or macroaneurysm $(\mathrm{p}=0.046)$, while showing a non-significant trend to improvement in wet AMD $(\mathrm{p}=0.10)$ and undifferentiated CNV $(\mathrm{p}=0.068)$. Mean and median length of follow-up were 19.2 months and 12 months, respectively, with a range of 1-63 months. Overall, 36/37 patients were seen at 3-month follow-up. Two patients with LP vision at 3-month interval were excluded from analysis. One patient was lost to follow-up after the 1-month interval and was analysed at 3-month interval using last observation carried forward.

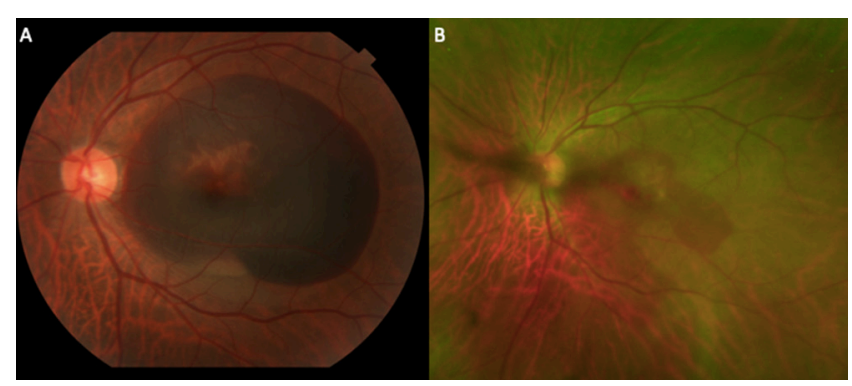

Figure 1 Fundus photography left eye (OS) of a patient at presentation (A) demonstrating dense submacular haemorrhage with foveal distortion and central pigment epithelial detachment (PED). Magnified widefield image (B) 3 weeks post-operative demonstrating resolution of dense submacular haemorrhage and restoration of foveal contour, with mild remaining flat haemorrhage.

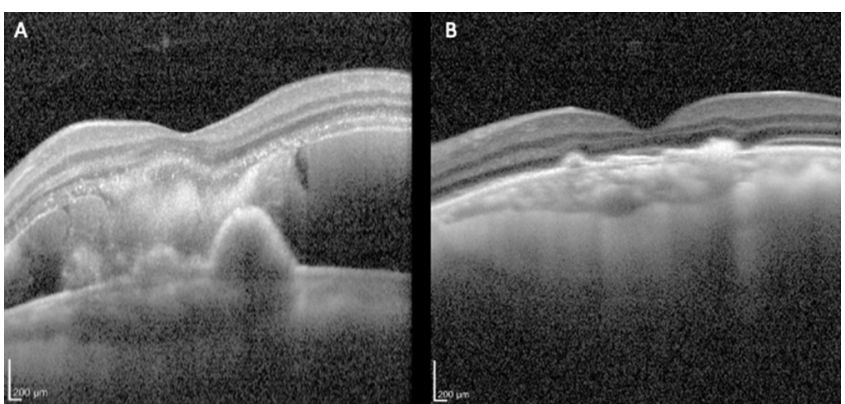

Figure 2 SD-OCT OS, before (A) and after (B) intervention demonstrating resolution of macular haemorrhage with good foveal contour, and collapsed pigment epithelial detachment with patchy overlying ellipsoid zone dropout. SD-OCT, spectral domain-optical coherence tomography.

Preoperative OCT data was available for $24 / 37$ patients. Mean preoperative central subfield thickness on OCT was $512.2 \mu \mathrm{m}$ for predominantly sub-RPE and $648.2 \mu \mathrm{m}$ for predominantly subretinal haemorrhage, with no difference in BCVA $(p=0.48)$. Comparison of BCVA by underlying diagnoses was not significant using one-way ANOVA at month $1(\mathrm{p}=0.98)$ or month $3(\mathrm{p}=0.60)$.

\section{DISCUSSION}

Acute, fovea-involving, dense submacular haemorrhage may result from a variety of underlying disease processes and result in profound vision loss if untreated. These results suggest some visual improvement following PPV with subretinal IPA and pneumatic displacement in most patients, regardless of the cause of SRMH. While prior studies have generally been limited to exudative AMD, this study includes all patients with SRMH regardless of underlying diagnosis. Prior wet AMD series have demonstrated modest visual recovery, anatomic success and relatively few adverse events. ${ }^{7-11}$ Some natural history studies have suggested worse outcomes of SRMH in patients with $\mathrm{CNV}$, while interventional studies have demonstrated improved outcomes in patients continuing to receive anti-VEGF treatments post-operatively. ${ }^{4}$ This

Table 2 Median visual acuities at baseline and intervals of interest, and post-operative complications. 'Other' includes glaucoma, corneal blood staining and pthisis.

\begin{tabular}{ll}
\hline Results & Snellen, LogMar (p value) \\
\hline $\begin{array}{l}\text { Median vision } \\
\text { Pre-op }\end{array}$ & $20 / 2000 ; 2.00$ \\
\hline Month 1 & $20 / 347 ; 1.24(<0.01)$ \\
\hline Month 3 & $20 / 152,0.88(<0.01)$ \\
Complications N (\%) & \\
\hline Vitreous haemorrhage & $5(13.5)$ \\
Recurrent haemorrhage & $4(10.8)$ \\
\hline RRD & $3(8.1)$ \\
Other & $3(8.1)$ \\
\hline
\end{tabular}

$\mathrm{RRD}$, rhegmatogenous retinal detachment. 
would suggest that it is prudent to continue anti-VEGF treatment postoperatively in patients with CNV.

Visual outcomes in our patients are like those previously reported in several exudative AMD studies, ${ }^{7811}$ and had greater improvement than in others. ${ }^{9}{ }^{10}$ One study by Gonzalez-Lopez et al reported slightly better outcomes with intervention earlier than 8 days, worse outcomes by area of haemorrhage and a similar overall final BCVA to this cohort at roughly 12 -month follow-up. ${ }^{12}$ More than half of our patients gained vision, with $41.7 \%$ of patients gaining significant vision of 3 or more lines at 3 months (figure 1A,B). Complications following intervention included vitreous haemorrhage, recurrent submacular haemorrhage, rhegmatogenous retinal detachment, glaucoma, corneal blood staining and phthisis, equal to or less frequent than previous reports. ${ }^{7-9} 11$ The mean and median times to surgery were 14.6 and 11 days, respectively, usually due to delay by patients in seeking evaluation, or referral delay from outside institutions in the community. Whether future studies demonstrate correlation between earlier intervention and improved visual outcome remains to be seen.

While follow-up was variable long term, it was excellent for our primary intervals of interest, with $36 / 37$ patients followed to 3 months. Given that many of these patients will likely continue to gradually lose vision due to the natural course of their diseases, a more conservative follow-up period was considered appropriate when evaluating results of surgical intervention alone. We did not analyse the BCVA at 1-month follow-up for our secondary outcomes since presence of intraocular gas will greatly limit interpretation. Additionally, a 3-month follow-up was chosen because of prior studies published with positive results at this interval, and this shorter interval limits confounding from usual progression of disease. ${ }^{8911}$ Of the patients diagnosed with 'undifferentiated CNV', 4/8 were suspected to have PCV but either lacked sufficient preoperative and postoperative testing (fluorescein angiography and/or indocyanine green angiography), or else had equivocal testing. Since the referral pattern of the institution includes a walk-in 'eye emergency department', a significant proportion of these patients were first-time visits, and have no data prior to acute SRMH including baseline vision prior to the event.

Neither anatomic location nor height of haemorrhage as determined by preoperative OCT were found to be of significant prognostic value, though this study is likely underpowered to find such an effect (figure 2A,B). Preoperative OCT was often not available for patients referred from outside institutions or satellite facilities. From our total patient cohort, 24/37 had preoperative OCT available. A large, retrospective study by Treumer et al also reported no correlation between preoperative height of haemorrhage on OCT, with a total of 132 eyes having a very similar final BCVA of $1.0 \log$ MAR compared with 1.09 logMAR in this cohort. ${ }^{13}$ Our other secondary outcome, a subgroup analysis of the relationship VA to underlying diagnosis, failed to reveal any statistical preference. This suggests that this treatment may be a viable option for all causes of dense, fovea-involving SRMH, regardless of aetiology. Caution is advised in interpreting these results, however, due to small numbers within subgroups. While this study lacks sufficient preoperative baseline VA data, it is likely that patients with poor baseline VA may expect more limited improvement. Common reasons for missing baseline acuity included first time presentation to the eye emergency department without history of evaluation, or referral from outside facility without available historical data. Of the 14 patients with baseline VA data available, the worst preoperative VA documented was 20/200 in two patients (range 20/20 to 20/200). Both patients worsened to counting fingers vision at 3 months, and did not improve at final follow-up.

The strengths of this study are that it includes a relatively large and diverse group of aetiologies with a common diagnosis, compared with prior studies limited solely to patients with exudative AMD. While we were not able to find significant differences in outcome between disease groups, a prospective, randomised study in the future may detect differences which will help predict expected patient outcomes. In concordance with natural history studies, we hypothesise that patients without underlying $\mathrm{CNV}$ (proliferativediabetic retinopathy, vein occlusion, macroaneurysm, among others) may ultimately have a better prognosis. Retrospective studies in populations with variable follow-up may be misleading and subject to a 'loss to follow-up' bias. ${ }^{14}$ The focus on interval data reported proportions of patients achieving specific visual gains at specific points postoperatively, allowing us to avoid the impact of variable follow-up. Additionally, for real-world, retrospective data of this nature, contiguous interval windows allow a more rigorous way to classify follow-up intervals. Nonetheless, several limitations of this study are important and must be considered. The retrospective and non-randomised nature of the patients included may be subject to a form of selection bias. Patients with less severe presentations may have been managed with conservative measures such as intravitreal injections, while patients with the more severe haemorrhage and poorer prognosis may have been deemed surgical candidates. If this were the case, patients would potentially be negatively skewed toward those with worse haemorrhages at presentation. In addition, given the referral pattern at our facility, we often did not have preoperative imaging or baseline VA data at time of presentation. Possibly, in some of those patients with unknown baseline vision, postoperative change interpreted as improvement may have regression to their presurgical acuity. Considering prior natural history studies, as well as our own longitudinal experiences, the authors feel that this data is compelling, and suggests that most patients with such a vision-threatening condition derive some benefit from this form of surgical intervention. 
CONCLUSION

PPV with subretinal tPA and pneumatic displacement of dense submacular haemorrhage appears to have significant benefit in promoting visual recovery, though long-term vision maintenance may depend on underlying pathology. Given the prospect of permanent severe vision loss, its notable that $61 \%$ of patients experienced some form of vision improvement, with $42 \%$ gaining three or more lines in vision at 3 month follow-up. Neither location of haemorrhage based on OCT imaging, nor the underlying pathophysiology of the SRMH, were predictive of visual outcome, however, a larger randomised study may better differentiate expected outcomes among these subgroups. While most patients with CNV will require continued anti-VEGF therapy, it is unclear whether this produces further visual recovery following displacement of dense SRMH. Adverse events occurred in some of the patients within this cohort, like prior reports, raising concerns about the importance of careful patient selection. Expectations of potential benefits and potential risks of the intervention must be carefully discussed with the patient during informed consent, particularly in cases of monocularity, high-risk medical conditions and poor baseline vision in the affected eye.

Contributors The following authors were responsible for data acquisition, analysis and manuscript drafting: CSW, NM, CYW, AB. The following authors were responsible for manuscript drafting and as content guarantors: AAD, RBR. All authors contributed significantly to the preparation of this manuscript.

Funding CSW is the recipient of a research grant from the Manhattan Eye Foundation.

Competing interests RBR reports consulting fees from Optovue, BoehringerIngelheim, NanoRetina, Bayer, Regeneron, CellView, and Astellas. He holds patents with Optovue and has a personal financial interest in Opticology and Guardion Health. None of the other authors report any relevant conflicts or proprietary interest in the results of this study.

Patient consent for publication Not required.

Provenance and peer review Not commissioned; externally peer reviewed.

Data availability statement Data are available upon request

Open access This is an open access article distributed in accordance with the Creative Commons Attribution Non Commercial (CC BY-NC 4.0) license, which permits others to distribute, remix, adapt, build upon this work non-commercially, and license their derivative works on different terms, provided the original work is properly cited, appropriate credit is given, any changes made indicated, and the use is non-commercial. See: http://creativecommons.org/licenses/by-nc/4.0/.

ORCID iD

Carl S Wilkins http://orcid.org/0000-0002-5289-9586

\section{REFERENCES}

1 Glatt $\mathrm{H}$, Machemer R. Experimental subretinal hemorrhage in rabbits. Am J Ophthalmol 1982;94:762-73.

2 Toth CA, Morse LS, Hjelmeland LM, et al. Fibrin directs early retinal damage after experimental subretinal hemorrhage. Arch Ophthalmol 1991;109:723-9.

3 Avery RL, Fekrat S, Hawkins BS, et al. Natural history of subfoveal subretinal hemorrhage in age-related macular degeneration. Retina 1996;16:183-9.

4 Berrocal MH, Lewis ML, Flynn HW. Variations in the clinical course of submacular hemorrhage. Am J Ophthalmol 1996;122:486-93.

5 de Silva SR, Bindra MS. Early treatment of acute submacular haemorrhage secondary to wet AMD using intravitreal tissue plasminogen activator, C3F8, and an anti-VEGF agent. Eye 2016;30:952-7.

6 Jain S, Kishore K, Sharma YR. Intravitreal anti-VEGF monotherapy for thick submacular hemorrhage of less than 1 week duration secondary to neovascular age-related macular degeneration. Indian J Ophthalmol 2013;61:490-6.

7 Haupert CL, McCuen BW, Jaffe GJ, et al. Pars plana vitrectomy, subretinal injection of tissue plasminogen activator, and fluid-gas exchange for displacement of thick submacular hemorrhage in agerelated macular degeneration. Am J Ophthalmol 2001;131:208-15.

8 Olivier S, Chow DR, Packo KH, et al. Subretinal recombinant tissue plasminogen activator injection and pneumatic displacement of thick submacular hemorrhage in age-related macular degeneration. Ophthalmology 2004;111:1201-8.

9 Chang W, Garg SJ, Maturi R, et al. Management of thick submacular hemorrhage with subretinal tissue plasminogen activator and pneumatic displacement for age-related macular degeneration. Am J Ophthalmol 2014;157:1250-7.

10 Novelli FJD, Preti RC, Monteiro MLR, et al. A new method of subretinal injection of tissue plasminogen activator and air in patients with submacular hemorrhage. Retina 2017;37:1607-11.

11 Sharma S, Kumar JB, Kim JE, et al. Pneumatic displacement of submacular hemorrhage with subretinal air and tissue plasminogen activator: initial United States experience. Ophthalmol Retina 2018;2:180-6.

12 González-López JJ, McGowan G, Chapman E, et al. Vitrectomy with subretinal tissue plasminogen activator and ranibizumab for submacular haemorrhages secondary to age-related macular degeneration: retrospective case series of 45 consecutive cases. Eye 2016;30:929-35.

13 Treumer F, Wienand S, Purtskhvanidze K, et al. The role of pigment epithelial detachment in AMD with submacular hemorrhage treated with vitrectomy and subretinal co-application of rtPA and anti-VEGF. Graefes Arch Clin Exp Ophthalmol 2017;255:1115-23.

14 Jabs DA. Improving the reporting of clinical case series. Am J Ophthalmol 2005;139:900-5. 